\title{
X-Ray Spectra and Time Variability of Active Galactic Nuclei
}

Richard F. Mushotzky

FEBRUARY 1984

National Aeronautics and

Space Administration

Goddard Space Flight Center

Greenbelt, Maryland 20771 
$X$-RAY SPECTRA AND TIME VARIABILITY OF ACTIVE GALACTIC NUCLE I 1

\author{
Richard F. Mushotzky \\ Laboratory for High Energy Astrophysics \\ NASA/Goddard Space Flight Center \\ Greenbelt, Maryland 20771
}

\title{
ABSTRACT
}

The X-ray spectra of broad line active galactic nuclei of all types (Seyfert I's, NELG's, Broadline radio galaxies) are well fit by a power law in the $.5-100 \mathrm{keV}$ band of mean energy slope $\alpha=.68 \pm .15$. There is, as yet, no strong evidence for time variability of this slope in a given object. The constraints that this places on simple models of the central energy source are discussed. BL Lac objects have quite different $X$-ray spectral properties and show pronounced $X$-ray spectral variability.

On time scales longer than 12 hours most radio quiet $A G N$ do not show strong, $\Delta I / I>.5$, variability. The probability of variability of these AGN seems to be inversely related to their luminosity. However characteristic timescales for variability have not been measured for many objects. This general lack of variability may imply that most AGN are well below the Eddington limit. Radio bright $A G N$ tend to be more variable than radio quiet AGN on long, $\tau \geqslant 6$ month, timescales.

${ }^{1}$ Talk given at Texas Meeting on Relativistic Astrophysics, Austin, Texas, 1982 and IAU/COSPAR Meeting on High Energy Astrophysics and Cosmology, Rojen, Bulgaria. 


\section{INTRODUCTION}

It is currently popular to believe that the $X$-rays from active galactic nuclei are produced in or near the physical region where the energy in these objects is released (see Rees, Begelman and Blandford 1981 for a recent discussion). If this is true, study of the $X$-ray emission from $A G N^{2}$ is a direct probe of the "infernal machine".

The data presented in this talk are primarily from spectroscopic experiments on the HEAO-1 and HEAO-2 (Einstein Observatory) X-ray astronomy satellites. These experiments covered the energy range from 2-100 keV (HEAO-1) and .5-4.5 $\mathrm{keV}$ (HEA0-2) and were capable of high accuracy spectroscopic and "photometric" measurements. The energy resolution in the $2-100 \mathrm{keV}$ band is roughly $16 \%$ or $1.1 \mathrm{keV}$ at $6 \mathrm{keV}$ and in the $.5-4.5 \mathrm{keV}$ it is $160 \mathrm{eV}$ or $8 \%$ at $2 \mathrm{keV}$. Both satellites had relatively short 1 ifetimes--HEA0-1 lived 17 months (Aug 1977 Jan 1979) and HEA0-2 lived 30 months (Nov 1978 - May 1981).

The collecting areas of these X-ray "telescopes" are rather small by radio or optical standards. HEAO-1 was equivalent to a 6.3" telescope and HEA0-2 to only a $3^{\prime \prime}$ telescope. However the high quantum efficiency $(>50 \%)$ of $X$-ray detectors helps to offset some of the difficulties. It is also useful to remember that the $X$-ray flux from $A G N$ is, in general, quite low. The weakest sources discussed here have a 2-10 kev flux of $-1 \times 10^{-3}$ photons $/ \mathrm{cm}^{2} \mathrm{sec}$ which is equivalent to a 22 magnitude object in the optical. (So to give the optical analog to $X$-ray spectroscopy try to imagine doing the optical spectrum of a 22 mag quasar with a 6" telescope!) (Of course the $X$-ray photons have - 1000 times as much energy so if we were comparing energy instead of photon flux the equivalent magnitude would be $-15 \mathrm{mag}$, still quite low).

2 In this paper the term active galactic nucleus (AGN) refers to the total assembly of objects whose nucleus emits "non-thermal" radiation. That is, the term AGN includes quasars, BL Lac objects, Sey'fert I's, broadline radio galaxies, etc. It is not clear, at present, if Seyfert II's or narrow line radin galaxies are members. 
THE X-RAY CONTINUA

\section{A. Broad Line AGN}

\section{Energetics}

The energy contained in the $X$-ray band (for the purposes of this discussion .5-100 keV, the range over which we have data) is roughiy the same as that in most other well observed bands (optical, IR, IJV). This is a consequence of the well known fact that, broadly speaking, the electromagnetic spectra of AGN follow a $\nu^{-1}$ power law over at least 8 decades in frequency (Worrall et al. 1981). In somewhat more detai1 the IR-IJV "power law" continua of AGN can be well modeled by a $\nu^{-1.2}$ power law (Malkan and Sargent 1982; Wu, Boggess and Gul1 1983; Glass 1983; Glass, Moorwood and Eichendorf 1982) while the X-ray continua are well modeled by a $v^{-0.7}$ power law (Rothschild et al. 1983; Mushotzky 1980; this paper). Thus a composite continuum spectrum in the observable energy ranges is $F_{v}=A v^{-1.2}+B \nu-0.7 \mathrm{ergs} / \mathrm{cm}^{2} \mathrm{sec} \mathrm{Hz}$. The total observed range of the ratio $\log (A / B)$ is from 7.5 to 11 (Zamorani et al. 1981; Reichert et al. 1982). The width of the distribution of the ratio of optical $(912-10,000 \AA)$ to $X$-ray $(.5-100 \mathrm{keV})$ luminosity, $L_{o p} / L_{x}$, is from .1 to 25. "Radio loud" AGN tend to have a smaller expectation value $\left\langle\mathrm{L}_{o p} / L_{x}\right\rangle-.6$ while "radio quiet" objects have $\left\langle\mathrm{L}_{o p} / \mathrm{L}_{x}>-2\right.$.

If the $X$-ray spectrum for most objects continues out to - 1 MeV (Baity et al. 1981) then the $X$-ray luminosity is increased by a factor of -2.5 and $\left\langle L_{o p} / L_{x}\right\rangle$ decreases to - .25 for radio loud objects. If the "non-thermal" power law continues down to $-10 \mu$, without flattening, then $L_{o p}$ is also increased by a factor of 2.5. Therefore the observed continua have roughly the same energy in all the observed wavelength bands except the radio (since the radio-optical spectral slope is flatter than 0.9 the radio, $10^{6}-10^{13} \mathrm{~Hz}$, has less energy than the optical). If there is a major "missing" luminosity it must be in the $\gamma$-ray or extreme $U-V$ bands. Present $\gamma$-ray upper 7 imits are not sensitive enough (except for $3 C$ 273) to constrain the $\gamma$-ray luminosity. 


\section{Spectral Form}

The X-ray spectrum of almost all broad line AGN (Figure 1) (Seyfert I's, quasars, broad line radio galaxies) can be well described (Figure 2) by a power law in the .5-100 keV band of $\alpha-.65 \pm .15$ modified by the effects of cold material in the line of sight. This spectral form appears quasi-universal, independent of luminosity (at least in the range from $L_{x}-10^{42.5}$ to $10^{46} \mathrm{ergs} / \mathrm{sec}$ in the $2-10 \mathrm{keV}$ band), and independent of optical type or radio property for nearby, $z<.1$, objects.

In Table I I give the $X$-ray spectral index for broad line AGN from HEAO-1 A? (2-40 keV band), HEA0-1 A4 (15-100 keV) and, for high luminosity sources $\left(L_{x}>\right.$ $10^{43.5}$ ergs/sec), the Einstein SSS data (.5-4.5 keV) (Petre et al. 1984). Simple power law moriels (modified by the effect of absorbing material in the line of sight) almost always give a good (reduced $x^{2}<1.3$ ) fit to the data. For roughly half the sample (Mushotzky 1982) analysis of the HEAO-1 A2 data strongly prefers a power law fit to the data over other simple spectral forms such as optically thin isothermal bremsstrahlung. Within the limits of the present data the spectral index fit to individual sources is the same in all 3 energy bands and there is no evidence for curvature or change of form with energy. I therefore conclude that this simple, one parameter model, that fits the data from more than 30 sources over a factor of 200 in energy, is probably the "correct" model.

In addition to having a simple spectral form the $X$-ray spectra from AGN have a very narrow range in spectral index. If we fit a Gaussian to the distribution of spectral indicies (Figure 2) we find $\bar{\alpha}=0.7 \pm .19$. For the smaller sample of 13 objects for which the statistical uncertainty $\delta \alpha<.15, \bar{\alpha}=.65 \pm .17$. Thus the intrinsic width of the distribution function is very narrow, with a dispersion probably less than .1. In some sense then the X-ray spectrum of broad line AGN is a well defined signature of these objects.

While the available data are rather sparse it seems as if this spectral index in the 2-40 keV band does not change when the 2-10 keV flux changes (however there is evidence (Baity et a1. 1983) that there has been a spectral change at $E>60 \mathrm{keV}$ in NGC 4151 and perhaps in the $1-10 \mathrm{keV}$ range in 3C 120 (Halpern 
1982) during flux variability). There is no correlation of X-ray spectral index with luminosity (at least in the range $10^{42.5}<\mathrm{L}_{\mathrm{x}}{ }^{2-10}<10^{45.5}$ ergs/sec); nor with optical type (e.g. Seyfert I with $\bar{\alpha}=.68 \pm .21$, broad line radio galaxy (BLRG) $\bar{\alpha}=.80 \pm .14$ or narrow emission line galaxy (NELG) $\bar{\alpha}=$ $.74 \pm .13)$; nor with the presence or absence or radio $\left(\nu<10^{10} \mathrm{~Hz}\right.$ ) emission (that is the radio bright objects $3 C 120,111,382,390.3$, Cen-A, and 3C273 have the same mean spectral index as the "radio quiet" Seyfert I's); nor with the $X$-ray to optical luminosity ratio.

As a general conclusion, then, the $X$-ray spectral form of "broadline" AGN is an extremely stable feature independent of every other external, visible characteristic of the objects. It is thus quite surprising that BL Lac type objects (see sec IIB) have a quite different and variable X-ray spectral form.

\section{Simple Theoretical Models}

Many workers have noted that under several quite reasonable scenarios of the energy generation mechanism in active galaxies, the $X$-rays are created in the same region as the bulk of the energy. Models of the $X$-ray emitting region (see Lightman 1982 for a review) have tended to concentrate on either thermal-Compton emission from an accretion disk around a black hole (or its equivalent) or on synchrotron self-Compton emission from relativistic particles. This section of the review concentrates on the constraints that the $X$-ray spectrum can place on these simple models.

In Figure 3 we show the predicted power law spectral indices from the non-relativistic and ultra-relativistic thermal-Compton models. In these models low energy photons, presumably from cool regions of the accretion disk, are scattered up to the $X$-ray energy range by Compton interactions with hot thermal electrons. We see that, for the non-relativistic models, if the temperature is > $70 \mathrm{keV}$ (as seems likely from the observations (Rothschild et al. 1983)) then the models are well constrained; the optical depth to Compton scattering must be between 1 and 2 and the electron temperature must be less than $280 \mathrm{keV}$ to reproduce the observed mean power law slope. This model predicts that there should be a rollover in the $X$-ray spectrum at roughly $E$ - KT which, someday, might be observable. In these models (see Shapiro, 
Lightman and Eardley, SLE, 1976) the ratio of Comptonized luminosity to soft photon luminosity is $A=L_{e} / L_{S}-\left(e^{y}-1\right)$ (see Fig. 1 of SLE for more $\operatorname{detai1}$ ) where $k T_{\star}=k T / m_{e} c^{2}, \tau$ is the optical depth and $y=4 k T_{\star} \max \left(\tau, \tau^{2}\right)$. To fit the AGN spectra, $0.5<y<8$, so that the amplification, $A$, is greater than 5. Thus these models predict that there should be more luminosity in the $X$-ray band than in the softer energy bands. Since the bolometric luminosity of $A G N, L_{B o l}$, is 2-100 times that of the $X$-ray luminosity, $L_{x}$, these models cannot, simply, account for the major part of the observed luminosity however well they account for the $X$-ray spectrum. This is a problem with all thermal-Compton models (e.g. Takahara 1982; Guilbert and Fabian 1982). The relativistic models require $\tau<.1$, in order that the temperature not be extraordinarily well specified. [However, as Araki and Lightman (1983) point out, equilibrium plasmas at $k T>511 \mathrm{keV}$ are unlikely to exist in AGN because the maximum luminosity of such a plasma is less than $10^{43.5} \mathrm{erg} / \mathrm{sec}$ if the objects have a scale size less than $10^{15} \mathrm{~cm}(.4$ light day).] Also since the Comptonized bremsstrahlung luminosity is proportional to $\tau^{2}$ (Lightman 1982) such a low $\tau$ plasma is less luminous than the non-relativistic case. Thus in these models the $X$-ray luminosity can be of the same order as the bolometric luminosity. Takahara (1982) has shown that for $\tau=0.1$, A can he as small as 1.1 but is a sensitive function of parameters and can be much larger (up to - 100 in Takahara's models).

If the $X$-ray emission is produced hy the synchrotron self-Compton process (Jones, O'Dell and Stein 1974) then the slope of the $X$-ray spectrum $\alpha$ is directly related to the spectrum of the relativistic electrons $s$ by $2 \alpha+1=$ s. Also it is predicted that the slope of the Comptonized photons $(X-r a y s)$ should be the same as the synchrotron (radio-millimeter) photons. As Rothschild et a1. (1983) point out, and as is illustrated in Figure 4, there is a remarkable uniformity in the distribution of a amongst a wide variety of astrophysical radio sources. This implies that for the cosmic ray electrons in the disk of spiral galaxies, in the cores of radio quasars, in double radio sources etc. that $\langle s\rangle-2.3 \pm .30$ and thus one predicts $\langle\alpha\rangle-.65 \pm .15$. This value and its intrinsic spread are extremely similar, within measurement error, to the X-ray spectra of broad line AGN. If the mean and variance of two quantities are the same it is highly likely that these quantities are the same. I thus consider this homology a strong argument for the origin of the 
$X$-ray flux as Compton scattering off a relativistic electron population of spectral index 2.3 .

This model also predicts that the $X$-ray spectrum should steepen at an energy $E_{B}^{-}-\gamma^{2} E_{B}$ where $E_{B}$ is the observed spectral steepening energy in the far IR and $\gamma$ is the relativistic factor for the electrons. For "typical" sources (Jones, O'Dell and Stein 1974) $\gamma=100-1000$ and $E_{B}=.1-.01$ eV so one predicts $E_{B}^{\prime}-1-10 \mathrm{MeV}$, consistent with observations of the $X$ and $\gamma$-ray background (Kazanas and Protheroe 1983). If the particles are more or less continously injected then the slope of the optical-UV spectrum should be $\alpha^{-}-\alpha_{x}+1 / 2$ or $\alpha^{-}-1.2$ as is consistent with most available data (Glass 1982; Malkan and Sargent 1982).

Of course this model does not specify the origin of the electron distribution or the energy. If this lies in shock acceleration (Ellison 1982; Blandford and Ostriker 1979) then the Mach number of the shock $M$ is related to the spectral index of the particles $s$ by $s=2\left(M^{2}+1\right) /\left(M^{2}-1\right)$, if the gas is adiabatic. For $s=2.4, M \approx 3.2$. The lower limit on $s=2.1$ from the distribution of $\alpha(\alpha-.7 \pm .15, \alpha>.55)$ would indicate $M \leq 6$.

A further prediction of this model is that the sources must be, relatively speaking, large. If sources were too small they would rapidly lose energy via Compton scattering. Roughly speaking, the physical source size $R$ should be related in a linear fashion to luminosity. Thus more luminous sources should be larger. For example, for $L=10^{44} \mathrm{erg} / \mathrm{sec}$, $\mathrm{R}$ should be $-10-100$ light days, much larger than black hole accretion disk models would predict (see Kazanas and Protheroe 1983).

B. BL Lac Objects and "Jets"

As implied in the previous section the X-ray spectrum of BL Lac objects is quite different from that of the broad line AGN's. It is quite interesting that the radio-optical selection criteria (no lines vs. broad lines, polarized vs. unpolarized, etc) should have a definite effect on the $X$-ray spectrum. 


\section{Energetics}

The total electromagnetic spectrum of a BL Lac object does not look much different from that of other AGN (Urry and Mushotzky 1982; Maraschi, Tanzi and Tarenghi 1983). However because of the frequencies at which the various bends in the spectrum occur and the ratio of soft to hard $x$-rays (see next section) most of the energy in BL Lacs probably lies in the soft $X$-ray-extreme ultra-violet band. In general BL Lacs have a high ratio, compared to Seyfert galaxies, of $1 \mathrm{keV}$ to optical flux but there is a large range (Schwartz and $\mathrm{Ku}$ 1983).

\section{Spectrum}

The X-ray spectrum of BL Lac objects (see Worrall et al. 1981) is characterized by its variability. In general the spectrum can be described by two components. The low energy component, which is always present, is considerbly steeper than that in Seyfert I's, $\alpha>1.0$. While the data can usually be well fit over a narrow energy range by a power law, the index can vary (contrary to the situation in Seyfert I's) from observation to observation. Also, quite often, (Urry and Mushotzky 1982; Urry et al. 1981) the ultraviolet and soft $X$-ray spectral indices are similar and the data is consistent with a constant (or slightly steepening spectral form) power law from the UV to soft X-ray. However there are exceptions such as MK180 (Mufson et al. 1982) where the $X$-ray emission lies above an extension of the UV continuum or PKS0735+178 (Bregman et a1. 1983) where the $X$-rays lie considerably below an extension of the UV power law.

The high energy component, which is not always present (Mushotzky et al. 1979), is usually quite flat, $\alpha<0.7$. However the narrow bandwidth over which it is observed (usually 4-15 keV) makes accurate determination of its slope difficult (Urry and Mushotzky 1982). Also for this reason it is difficult to estimate the amount of energy in the flat component. Because of the lack of data about the flat component (it is usually visible only above the bandpass of the Einstein imaging telecope), its range in spectral slope and flux is quite uncertain. 
The variability of the $X$-ray spectral form can be represented in two simple ways, 1) A change in the slope of the low energy component in the soft $x$-ray and 2) a change in the ratio of the hard to soft component. For example in MK421 the effective soft $X$-ray spectral index has changed from $\alpha-1.1$ to a 2.9 (Mushotzky et al. 1979) while in 3C 66 it changed from 1.1 to $\alpha \geq 2.5$ (Maccagni, Maccacaro and Tarenghi 1983). During the same "event" in MK 421 the hard component decreased in intensity by at least a factor of 10.

\section{X-Ray "Spectra" of Jets}

The detection of X-ray emission from jets in M87 (Schreier et al. 1982) and Cen-A (Feigelson et a1. 1981) allows one to compare the radio-X-ray optical properties of these objects with that of BL Lac objects. The spectrum of Knot $A$ in the $M 87$ jet (Schreier et al.) is very similar to the spectrum of PKS0548-322. In M87 the radio-optical spectral index $\alpha_{R O}=.55$, the IR index $\alpha_{1 R}-0.8$, optical index $\alpha_{0} \fallingdotseq 1.7$ and the $x$-ray optical index $\alpha_{0 X}=1.4$ while in 0548-322 the values are $\alpha_{R O}=.4, \alpha_{U V}=.8, \alpha_{0 X}=1.2$. Unfortunately, at present, this is the only jet for which a detailed comparison can be made. It thus seems that the observed electromagnetic spectrum of the jet in M87 is similar to that of at least some BL Lac objects. However it is also similar to that of radio bright QSO's. There is as yet no good idea of how (or if) it correlates with other continuum components.

\section{Theoretical Models}

As reviewed in Urry (1983) there are strong arguments in favor of attributing the total electromagnetic spectrum of BL Lacs to the presence of a relativistic jet at a small angle to our line of sight. While we will not go into detail here we can summarize the result by stating that in this interpretation the radio-optical radiation is due to optically "thick" synchrotron emission from a jet, the optical-UV-soft $X$-ray from optically thin synchrotron emission (with the effects of losses included) and the hard $X-r a y$ tail due to inverse Compton emission (Konigl 1981). 


\section{TIME VARIABILITY}

Another major constraint on tine nature of the central energy source is the nature of its time variability. If a major fraction of the observed luminosity varies on a timescale $\Delta \tau\left(\Delta \tau=(d \ell n L / d t)^{-1}\right)$ then a size scale $R$ - Ac $\Delta \tau$ can be set for the size of the emission region. Here $A$ depends on geometry and dynamics but if relativistic effects do not dominate $A$ is less than 3 (Jones, O'Dell and Stein 1974).

Black hole models predict, roughly, that $\Delta \tau-10 \mathrm{GM} / \mathrm{c}^{3}-50 M_{6} \mathrm{sec}$ where $M_{6}$ is the mass of the central object in units of $10^{6} M_{0}$. Since $M_{6}$ is expected to be in the range $0.1-1000$, (if the objects emit at -0.1 the Eddington limit), one might expect characteristic timescales from minutes to $-1 / 2$ day if the $X$-rays are directly associated with the black hole. The dynamical or thermal time scale for an accretion disk can be considerably longer and may, in fact, be the dominant time scale in the system. Such time scales are seen in the galactic black hole candidate cyg $X-1$. This object shows short, $\Delta \tau-10^{-3}$ sec, variability which using the above formula would be expected from a $10 M_{0}$ black hole. However most of the power in its variability occurs at longer timescales $\Delta \tau-1 \mathrm{sec}$. Scaling this longer time scale to the $10^{6}-10^{9} \mathrm{M}_{0}$ objects expected in AGN would give observed variability in the 1-1000 day range.

\section{A. Long Time Scales}

The HEAO-1 satellite scanned the entire sky $21 / 2$ times with an average exposure length of -4 days per source. This data allows us to look for variability on $1 / 2-11 / 2$ year timescale for all the sources detected by HEAO-1. For the brighter sources (> $6 \times 10^{-11} \mathrm{erg} / \mathrm{cm}^{2}$ in the $2-10 \mathrm{keV}$ band) it is also possible to look for variability on $1 / 2-4$ day time scale.

Of the 28 Seyfert I galaxies detected by HEAO-1, only 6 showed significant variability. That is, for only six sources was there flux inconsistent with a constant at the $93 \%$ confidence level $(1.80)$. If we raise the required significance level to $96 \%$ confidence (e.g. expect 1 out of 28 to be variable by chance) there are 5 variable sources. The strong sources would have had to 
vary by $\geq 30 \%$ and the weaker sources by $>50 \%$ to be detected as variable by this criteria. We thus conclude that for Seyfert I galaxies variability by > $50 \%$ on timescales from $1 / 2$ to $11 / 2$ years is uncommon.

The average luminosity of the variable sources, $\log \tau_{x}-43.16 \pm .47 \mathrm{ergs} / \mathrm{sec}$, is -.2 of that of the non-variable sources $\log \left[_{x}=43.91\right.$. If one includes the narrow emission line galaxies (Mushotzky 1982) whose X-ray luminosities are similar to that of low luminosity Seyfert I's one constructs Figure 5 . In this figure one can see that the probability of variability of radio-faint. emission line galaxies seems to be inversely related to their $X$-ray luminosity, at least on these long time scales. A similar result can be derived from the Ariel 5 data of Marshal1, Warwick and Pounds (1981). The situation for radio bright objects is quite different. Most of the radio bright AGN detected by HEAO-1 are variable. A similar result was obtained by Halpern (1982). Of the 13. objects in his sample more luminous than $10^{44}$ $\mathrm{erg} / \mathrm{sec}$, the 6 which are radio bright were variable, while only 2 of the 7 radio quiet sources varied. Thus, at a given $X$-ray luminosity, radio bright sources tend, on long time scales, to be more variable than radio quiet ones.

\section{B. Intermediate Time Scales}

There were 13 Seyfert I's bright enough in the HEAO-1 data to look for day to day X-ray variability. With the exception of NGC 6814 and NGC 4151 none of the sources showed variability $>60 \%$ on timescales from $1-4$ days with most of the upper limits indicating < $30 \%$ variability. We thus conclude that intermediate term variability, 1-4 days is a rare occurence in the $X$-ray flux from Seyfert I galaxies. For the narrow emission line galaxies there were 5 of 17 observations that indicated variability. The amplitude of variability in all but one of these was $<50 \%$ and the timescales were $\geq 2$ days. In this sample of Seyfert I's and NELG's the only objects that showed evidence for variability on < 4 day timescales were low luminosity objects. We therefore conclude from this relatively sparse data that large amplitude, $\Delta I / I>.5$, variability on time scales from $1-4$ days is also rare. 


\section{Short Time Scales}

The HEA0-1 pointed data (Tennant et al. 1981; Tennant and Mushotzky (TM) 1983) has excellent sensitivity, $\Delta I / I$ - .I or better, to variability on timescales from 5-30,000 seconds. As shown by TM in a sample of 54 observations of 38 objects only one object showed variability on timescales less than three hours. Three objects, NGC 3227, NGC 4151 and MCG 5-23-16, showed evidence for variability consistent with a time scale of - 1 day. Thus rapid $X$-ray variability is also unusual in AGN. (For a more extensive discussion of these data see TM 1983).

This is not to say that such rapid variability is unknown. There are at least two well documented cases of large amplitude, $\Delta I / I>.5$, short

timescale, $\tau<3$ hours, variability known, NGC 6814 (Tennant et a1. 1981) and NGC 4051 (Marshall et al. 1983). These observations are very tantilizing. Why do these objects show the "expected" short term variability while none of the others do? We note that NGC 4051 and NGC 6814 are among the least luminous of all AGN. However we shall leave interpretation of this result for future theoretical speculation.

\section{Summary}

Contrary to most recent discussions (e.g. Pounds 1979) the HEAO-1 data show that large amplitude $\Delta I / I>.5$ variability of the $X$-ray flux from $A G N$ is rare on all time scales from seconds to years. For a few objects (e.g. NGC 4151) the characteristic time scale is - 1 day (but even for NGC 4151 there were only two "l-day flares" in 29 days of observation). It is possible (e.g. MK 509) that quite a few objects may vary on a timescale of weeks but much better data is needed (TM 1983).

It is unclear what constraints the non-detection of variability place on theoretical models. However it is clear that whatever the model it must not be very similar to those that successfully produce the short time scale variability in Cyg $x-1$. 
One obvious model (cf. TM 1983) would be one in which the X-rays come from a large volume e.g. $R \geqslant 1$ lightday $\left(2 \times 10^{15} \mathrm{~cm}\right)$, which is a requirement of most synchrotron self Compton (SSC) models. If one insists that the production of $X$-rays be associated with a massive blackhole then one is led to the conclusion that $M_{B H} \geq 10^{9} M_{0}$. Since for most of these objects the total luminosity is less than $10^{46} \mathrm{ergs} / \mathrm{sec}$ one can infer that these objects emit less than $10^{-3}$ of the Eddington luminosity. Such sub-Eddington accretion is consistent with stable flow (Krolik and London 1983). I find it interesting that, with the exception of NGC 6814, both the $X$-ray spectral and temporal behavior is consistent with SSC models. However consistency is not proof and clearly more detailed observations are necessary as well as models with more predictive power.

REFERENCES (review of literature finished Oct. 1983)

Araki, S., and Lightman 1983, Ap. J. 2069, 49.

Baity, W., et al. 1981, Ap. J. 244, 429.

Baity, W., Mushotzky, R., Worral1, D., Rothschild, R., Tennant, A., and Primini, F. 1983, Ap. J., in press.

Blandford, R., and Ostriker, J. 1978, Ap. J. (Letters) 221, L29.

Bregman, 1., et al. 1983, preprint.

Ellison, D. 1982, Ph.D. Thesis, Catholic University of America.

Feigelson, E., Schreier, E., Delvaille, J., Giacconi, R., Grindlay, J., and Lightman, A. 1981, Ap. J. 251, 31.

Glass, I. 1982, M.N.R.A.S. 197, 1067.

Glass, I., Moorwood, A., and Eichendorf, W. 1982, A \& A 107, 276.

Guilbert, P., and Fabian, A. 1982, Nature 296, 226.

Halpern, J. 1982, Ph.D. Thesis, Harvard University.

Jones, T., O'Dell, S., and Stern, W. 1974, Ap. J. 188, 353.

Lightman, A. 1982, pg. 143 in X-Ray Astronomy in the 1980's, ed. by S. Holt, NASA TM 83848.

Kazanas, D., and Protheroe, R. 1983, Nature 302, 228.

Krolik, J., and London, R. 1983, Ap. J. 267, 18.

Konigl, A. 1981, Ap. J. 244, 700.

Maccagni, D., Maccacaro, T., and Tarenghi, M. 1983, preprint.

Malkan, M., and Sargent, W.L.W. 1982, Ap. J. 254, 22. 
Maraschi, L., Tanzi, E., and Treves, A. 1983, preprint.

Marsha11, F., Holt, S.S., Mushotzky, R., and Becker, R. 1983, Ap. J. (Letters) 269, L31.

Marsha11, N., Warwick, R., and Pounds, K. 1981, M.N.R.A.S. 194, 987.

Mufson, S., et al. 1982, pg. 185, Advances in U1traviolet Astronomy: 4 years of IUE Research, ed. Y. Kondo, J. Mead and R. Chapman, NASA TM 2238.

Mushotzky, R., Boldt, E., Holt, S., and Serlemitsos, P. 1979, Ap. J. (Letters) 232, L17.

Mushotzky, R. 1982, Ap. J. 256, 92.

Petre, R., Mushotzky, R., Krolik, J., and Holt, S.S. 1984, Ap. J., in press. Pounds, K. 1979, Proc. Roy. Soc. London A 366, 375.

Pozdnyakov, L., Sobol, I., and Sunyaev, R. 1976, Soviet Astr. Letters 2, 55. Rees, M., Begelman, M., and Blandford, R. 1981, in 10th Texas Symposium on Relativistic Astrophysics, ed. R. Ramaty and F. Jones (New York: New York Academy of Sciences), p. 254.

Reichert, G., Mason, K., Thorstensen, J., and Bowyer, S. 1982, Ap. J. 260, 437.

Rothschild, R., Mushotzky, R., Baity, W., Gruber, D., Matteson, J., and Peterson, L. 1983, Ap. J. 269, 423.

Schreier, E., Gorenstein, D., and Feigelson, E. 1982, Ap. J. 261, 42.

Schwartz, D., and Ku, W.H. 1983, Ap. J. 266, 459.

Shapiro, S., Lightman, A., and Eardley, D. 1976, Ap. J. 204, 187.

Takahara, F. 1982, preprint.

Tennant, A., Mushotzky, R., Boldt, E., and Swank, J. 1981, Ap. J. 251, 15.

Tennant, A., and Mushotzky, R.F. 1983, Ap. J. 264, 92.

Urry, C.M., Mushotzky, R., Kondo, Y., Hackney, K., and Hackney, R. 1982, Ap. J. $261,12$.

Urry, C.M., and Mushotzky, R. 1982, Ap. J. 253, 38.

Urry, C.M. 1983, Ph.D. Thesis, Johns Hopkins University. Worrall, D., Boldt, E., Holt, S., Mushotzky, R., and Serlemitsos, P.J. 1981, Ap. J. 243, 53.

Wu, C.C., Boggess, A., and Gul1, T.R. 1983, Ap. J. 266, 28.

Zamorani, G., et al. 1981, Ap. J. 245, 357. 


\section{FIGURE CAPTIONS}

Figure la - The HEAO-1 2-40 keV spectra of 10 Seyfert I's. The $L_{x}$ value is the 2-10 keV X-ray luminosity.

Figure lb - The HEAO-1 2-40 keV spectra of 4 broad line radio galaxies.

Figure 2 - The spectral index distribution of emission line active galaxies.

Figure 3 - A plot of energy index, $\alpha$, versus $T_{\star}=k T / m c^{2}$ for a variety of optical depths, $\tau$. The dashed lines are for non-relativistic models, the solid line for relativistic models (Pozdnyakov, Sobol and Sunyaev 1976).

Figure 4 - Spectral index distributions for a variety of objects (see Rothschild et al. 1983 for references).

Figure 5 - Time variability on a 6 month scale vs. $L_{x}$. 
TABLE I: $\quad X$-RAY SPECTRAL PARAMETERS FOR BROAD LINE ACTIVE GALAXIES

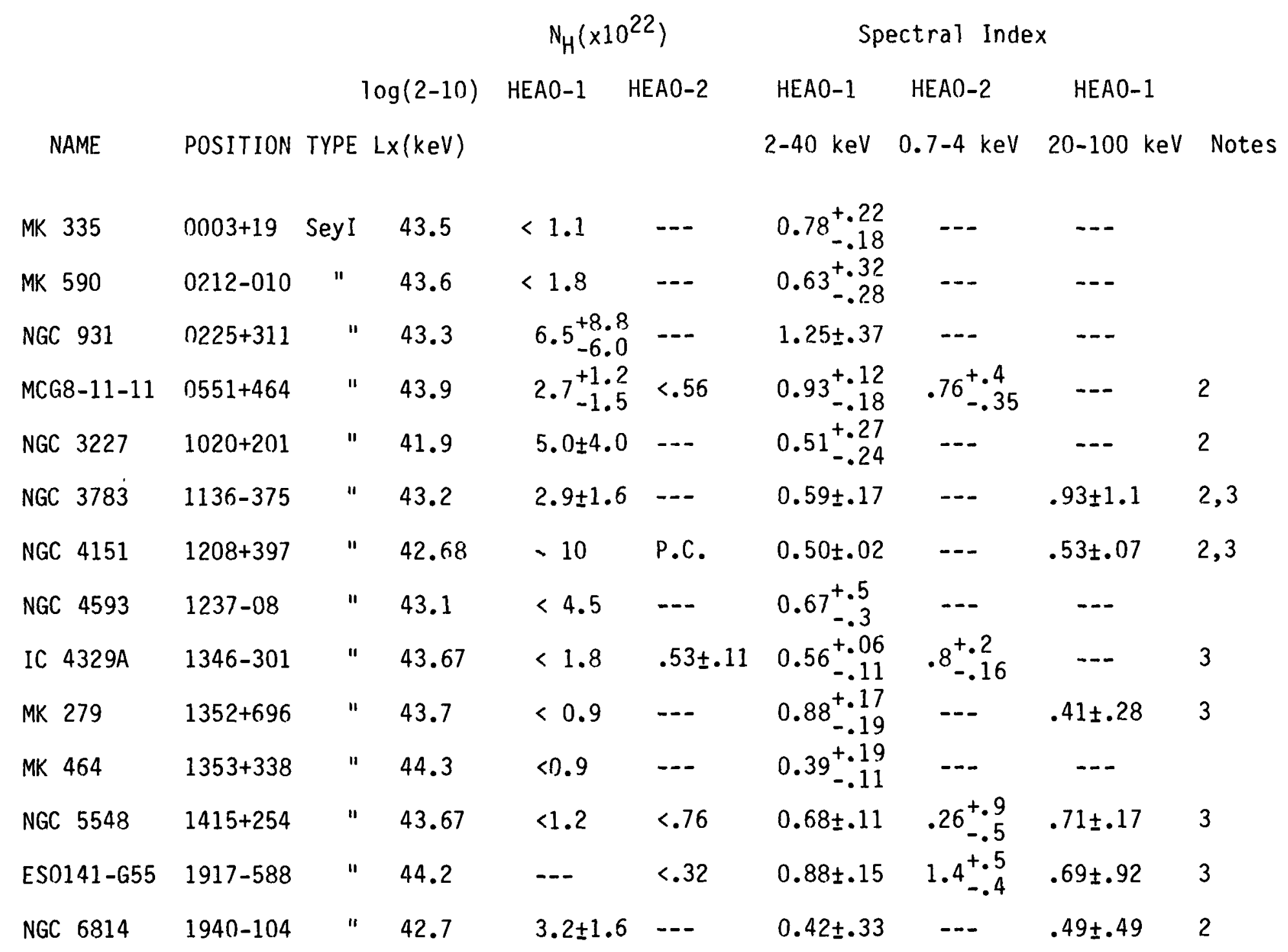




\begin{tabular}{|c|c|c|c|c|c|c|c|c|c|}
\hline MK 509 & 2041-109 & $"$ & 44.3 & $<0.54$ & $<.4$ & $0.63 \pm .10$ & $.93^{+.3}$ & $.47 \pm .26$ & 3 \\
\hline NGC 7213 & $2206-474$ & $"$ & 42.6 & $<1.2$ & -- & $\begin{array}{l}0.65^{+.30} \\
-.20\end{array}$ & --- & --- & 2 \\
\hline NGC 7469 & $2300+086$ & $"$ & 43.8 & $<0.55$ & $<.18$ & $0.78+.25$ & $.88 \pm .3$ & -- & \\
\hline$M C G-2-58-22$ & $2302-090$ & $"$ & 44.7 & $<0.90$ & $<.24$ & $0.55_{-.04}^{+.10}$ & $.53 \pm .25$ & --- & 3 \\
\hline NGC 526a & $0121-353$ & NELG & 43.65 & $<4.3$ & --- & $\begin{array}{r}0.52^{+.30}-.13\end{array}$ & $-\cdots$ & $\cdots$ & 2 \\
\hline NGC 2110 & $0549-075$ & $"$ & 43.16 & $\begin{array}{r}7.4^{+1.9} \\
-1.4\end{array}$ & --- & $0.80 \pm .15$ & -- & -- & 2 \\
\hline NGC 2992 & $0943-140$ & $"$ & 43.18 & $1.6 \pm .9$ & --- & $0.79 \pm .10$ & --- & -- & \\
\hline MCG $-5-23-16$ & $0945-310$ & $"$ & 43.34 & $1.7^{+1.0}-0.7$ & --- & $0.84 \pm .17$ & $.99 \pm .43$ & $\cdots$ & 2,3 \\
\hline NGC 5506 & $1410-030$ & $"$ & 43.0 & $\begin{array}{r}4.9^{+1.4}-1.6\end{array}$ & -- & $0.75 \pm .15$ & -- & -- & 3 \\
\hline NGC 7582 & $2315-426$ & $"$ & 42.6 & -- & $\cdots$ & $-.69 \pm .15$ & --- & --- & \\
\hline $3 C 111$ & $0415+379$ & BLRG & 44.7 & $<1.1$ & $<2.4$ & $\begin{array}{r}0.90^{+.22} \\
-.13\end{array}$ & $0.0_{-.40}^{+1.0}$ & --- & 2 \\
\hline $3 C 120$ & $0430+052$ & $"$ & 44.41 & $<1.6$ & $<.3$ & $0.72^{+.23}$ & $0.4^{+.3}$ & $.40 \pm .34$ & 1,3 \\
\hline $3 C 382$ & $1833+327$ & $"$ & 44.87 & $\begin{array}{r}2.7+2.7 \\
-2.6\end{array}$ & $<.39$ & $0.93^{+.32}-.28$ & $\begin{array}{r}0.1+.7 \\
-.3\end{array}$ & -- & \\
\hline $3 C 390.3$ & $1845+797$ & $"$ & 44.4 & $<4.9$ & -- & $\begin{array}{r}0.65^{+.50} \\
-.25\end{array}$ & $-\cdots$ & $.40 \pm .37$ & 2 \\
\hline Cen-A & $1322-928$ & $\begin{array}{l}\text { Radio } \\
\text { Gal }\end{array}$ & $42.6 !$ & -13 & -- & $0.62 \pm .04$ & --- & $0.63 \pm .04$ & 2,3 \\
\hline $3 C 273$ & $1226+023$ & QSO & 45.8 & $<.45$ & $<.11$ & $0.41 \pm .02$ & $.45^{+.20}-.13$ & $0.67 \pm .14$ & 2,3 \\
\hline $\begin{array}{l}1 \\
2 \\
3 \\
\text { P.C }\end{array}$ & $\begin{array}{l}\text { Possibly } \\
\text { Flux is va } \\
\text { Power law } \\
\text { = partia }\end{array}$ & $\begin{array}{l}\text { ariable } \\
\text { riable } \\
\text { signifi } \\
\text { lly cor }\end{array}$ & $\begin{array}{l}\text { spectr } \\
\text { (from } \\
\text { icantly } \\
\text { vered }\end{array}$ & $\begin{array}{l}-1 \text { data } \\
\text { eer fit }\end{array}$ & $\begin{array}{l}\text { only) on } \\
\text { than ther }\end{array}$ & $\begin{array}{l}6 \text { month ti } \\
\text { mal bremss }\end{array}$ & $\begin{array}{l}\text { ne scale } \\
\text { crahlung }\end{array}$ & & \\
\hline
\end{tabular}



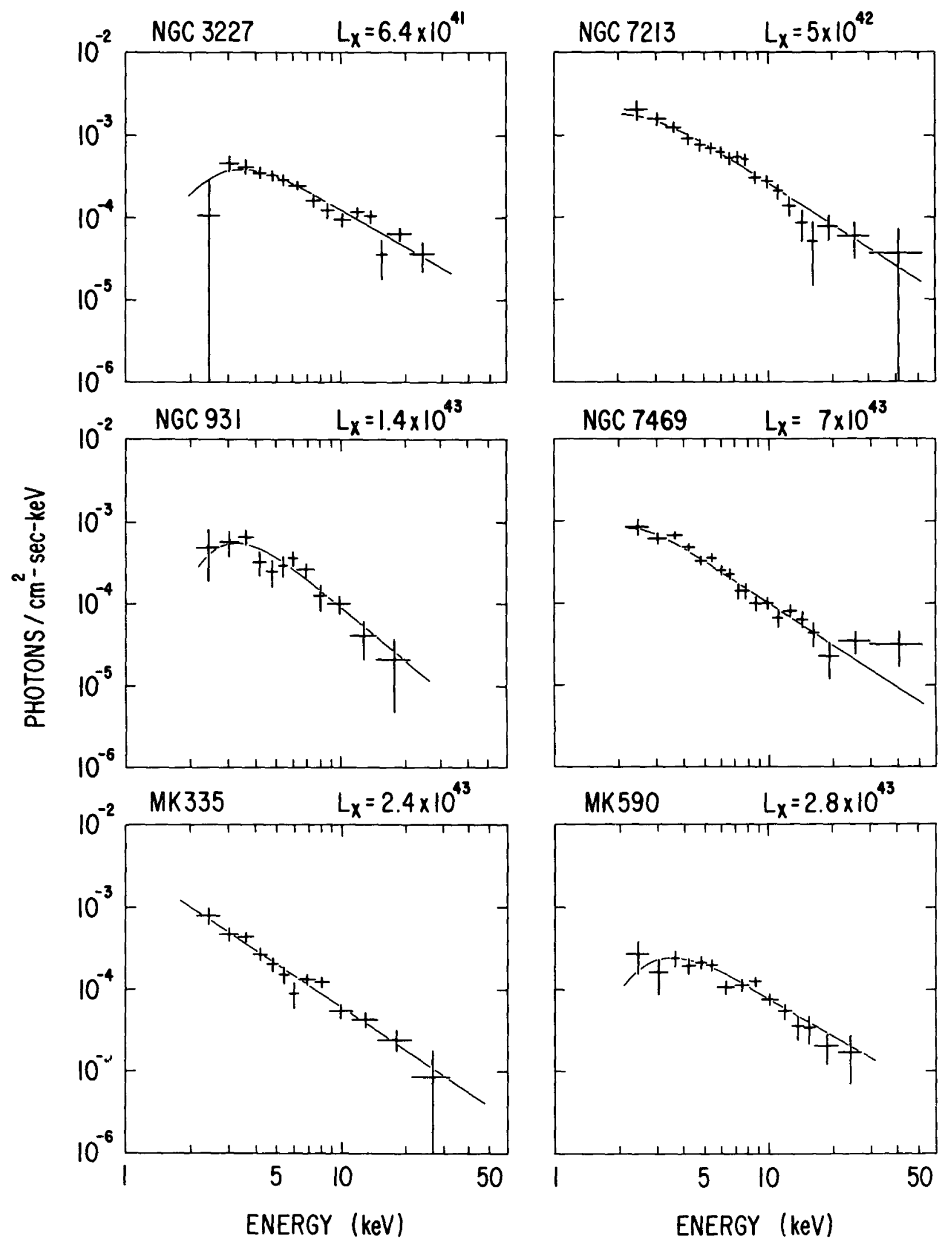

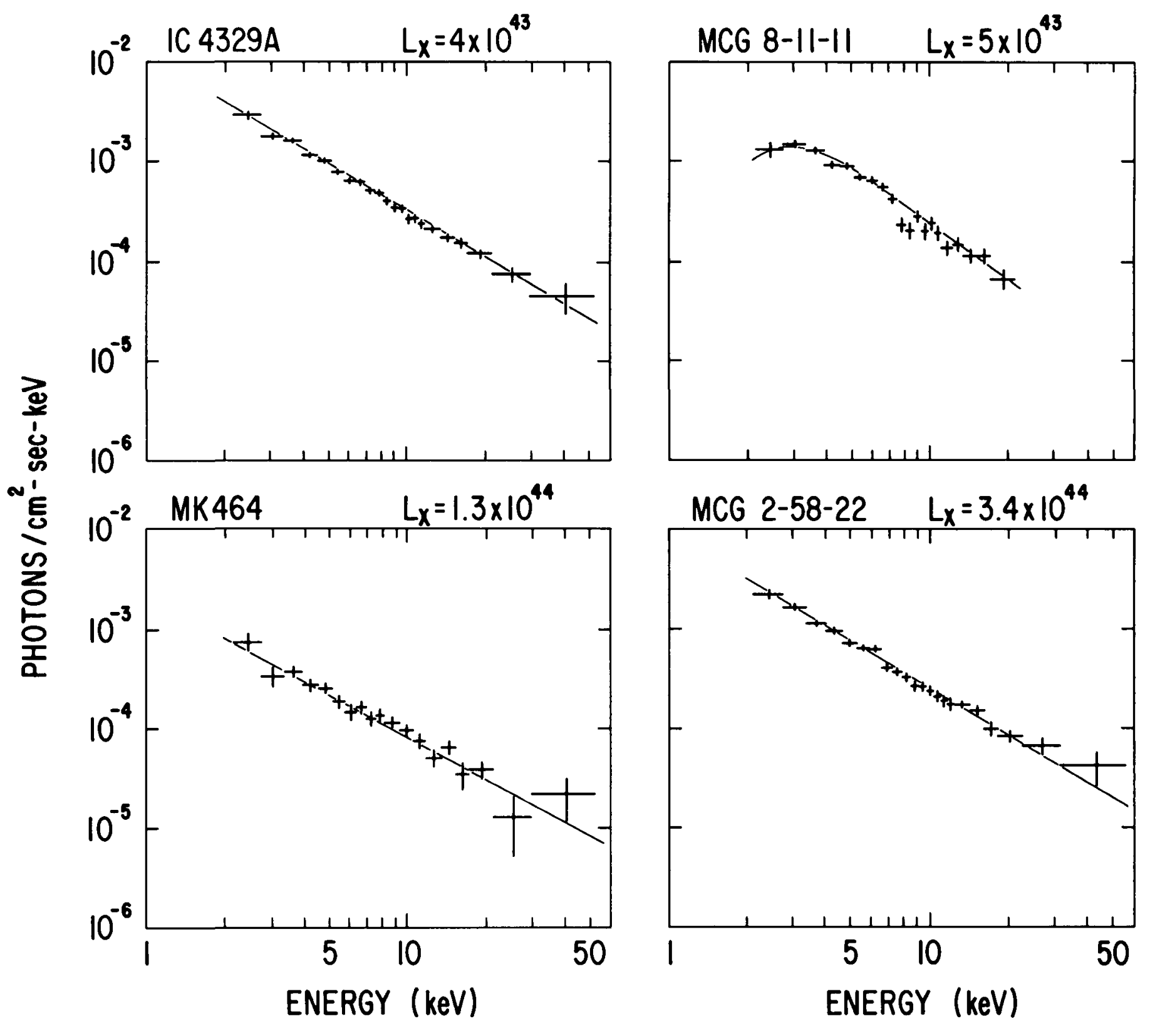


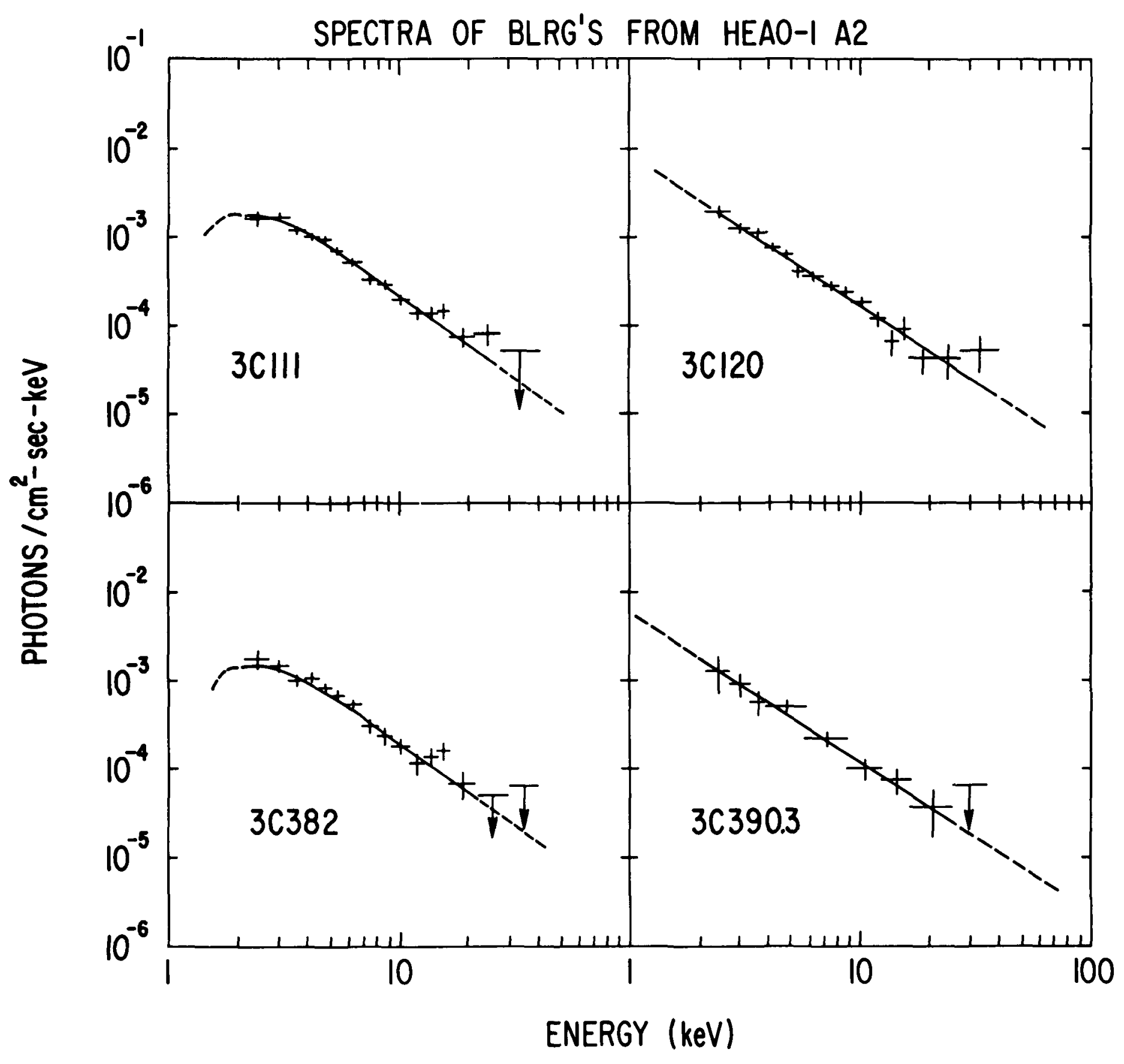




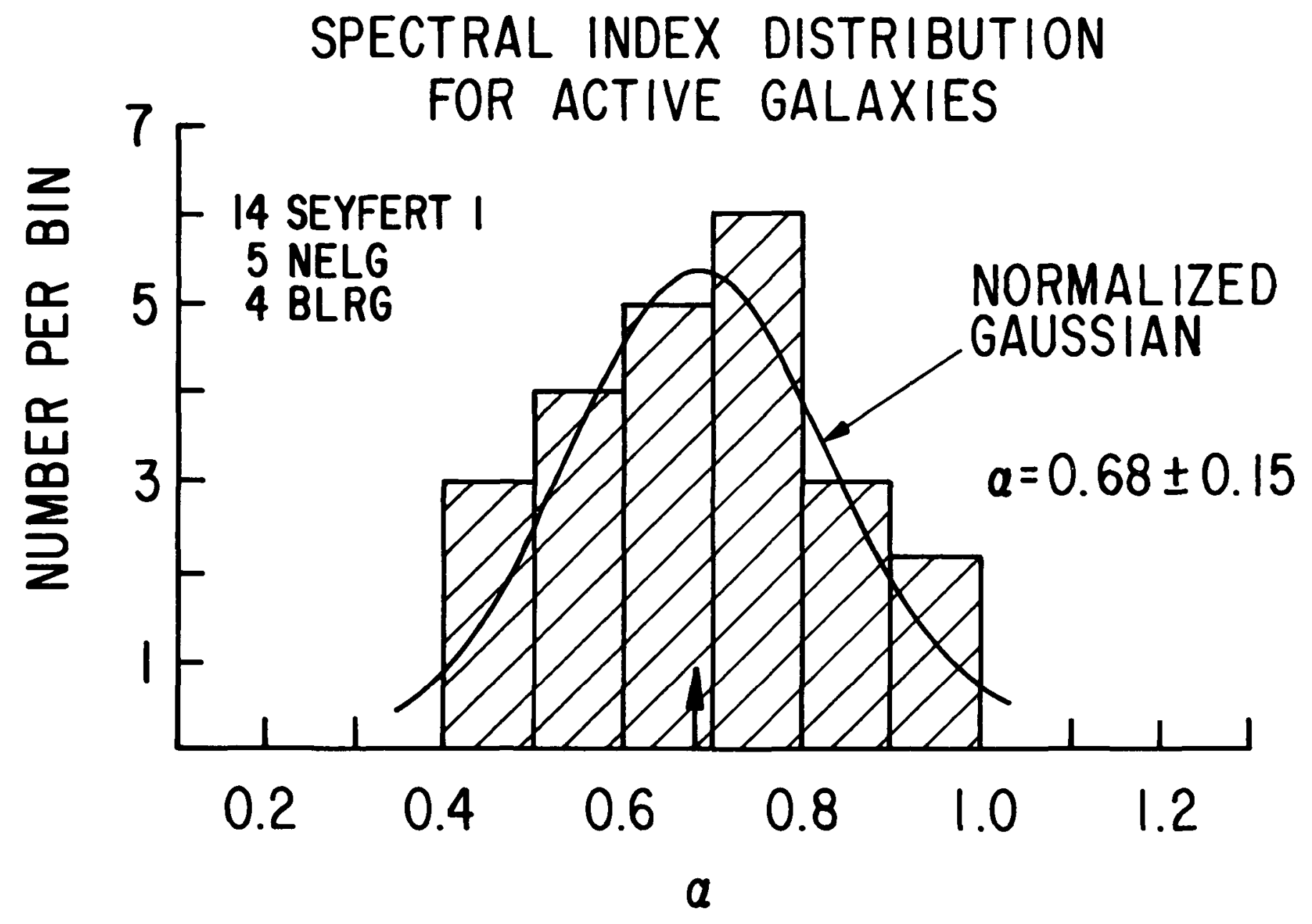


THERMAL COMPTON MODEL

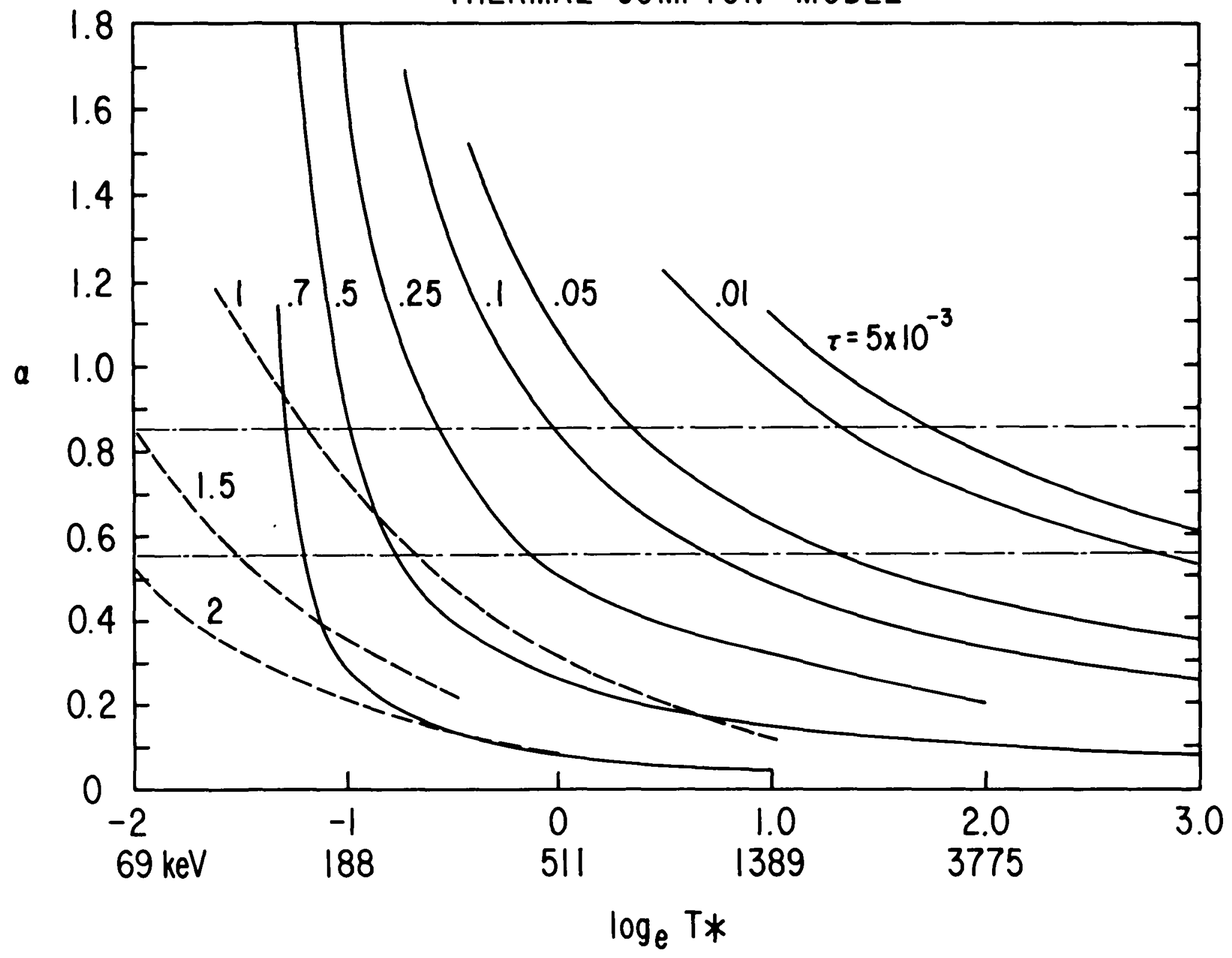




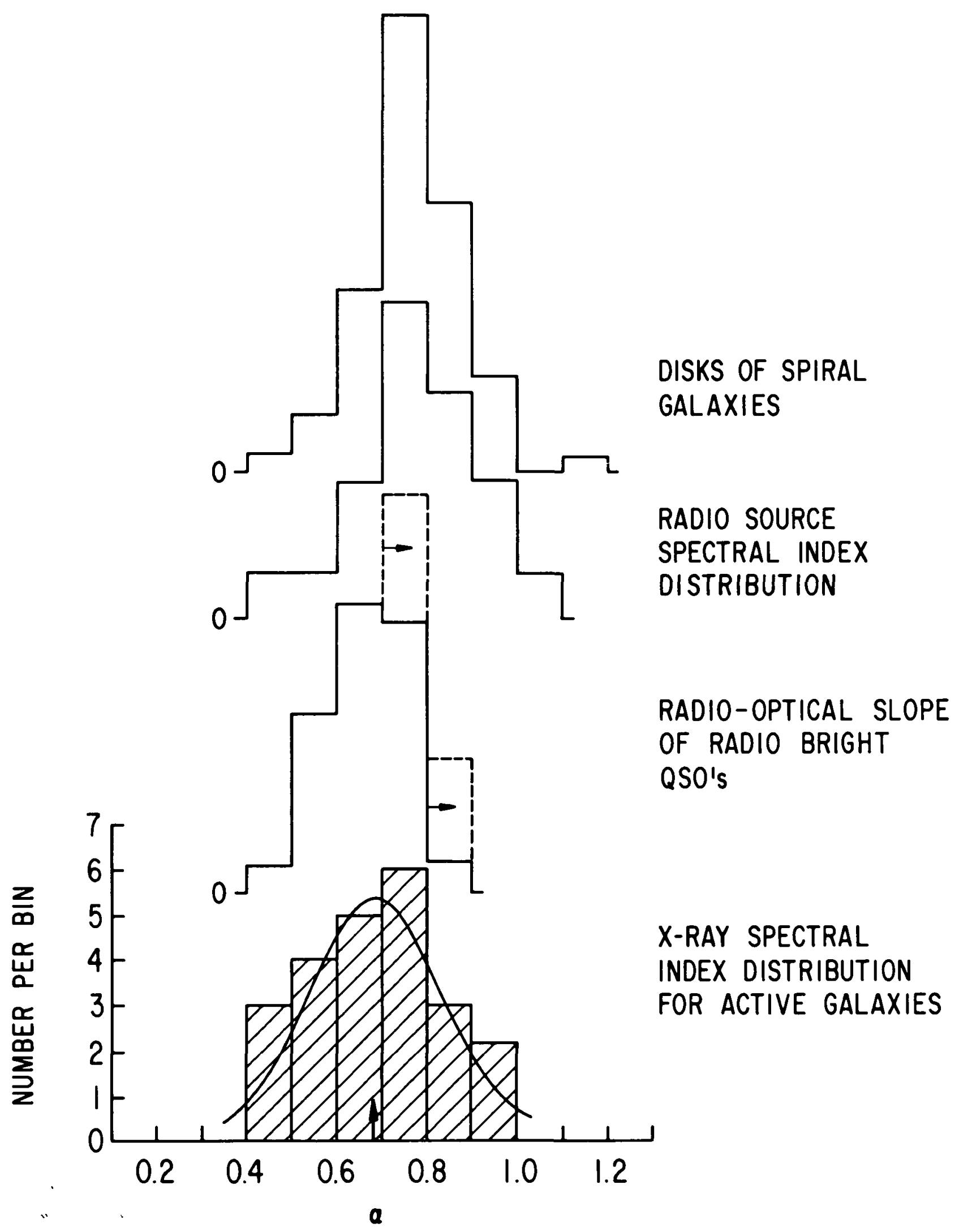




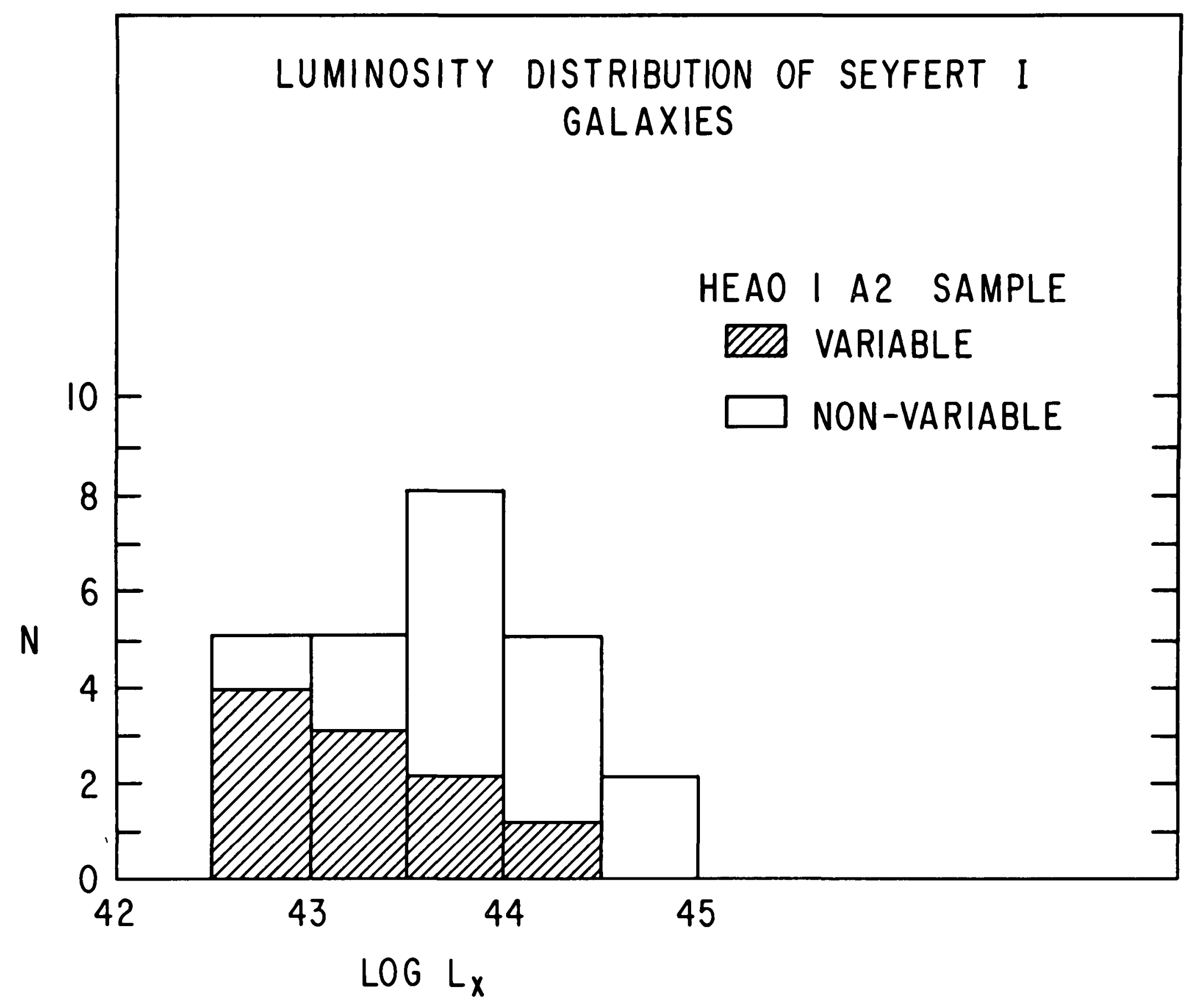


